\title{
Article \\ Giant Second Harmonic Generation Enhancement by Ag Nanoparticles Compactly Distributed on Hexagonal Arrangements
}

\author{
Alejandro Gómez-Tornero, Luisa E. Bausá and Mariola O. Ramírez *D \\ Departamento Física de Materiales, Instituto de Materiales Nicolás Cabrera and Condensed Matter Physics \\ Center (IFIMAC), Universidad Autónoma de Madrid, 28049 Madrid, Spain; \\ alejandrogomeztornero@gmail.com (A.G.-T.); luisa.bausa@uam.es (L.E.B.) \\ * Correspondence: mariola.ramirez@uam.es
}

Citation: Gómez-Tornero, A.; Bausá, L.E.; Ramírez, M.O. Giant Second Harmonic Generation Enhancement by Ag Nanoparticles Compactly Distributed on Hexagonal Arrangements. Nanomaterials 2021, 11, 2394. https://doi.org/10.3390/ nano11092394

Academic Editor: Giuseppe Chirico

Received: 28 July 2021

Accepted: 10 September 2021

Published: 14 September 2021

Publisher's Note: MDPI stays neutral with regard to jurisdictional claims in published maps and institutional affiliations.

Copyright: (c) 2021 by the authors. Licensee MDPI, Basel, Switzerland. This article is an open access article distributed under the terms and conditions of the Creative Commons Attribution (CC BY) license (https:// creativecommons.org/licenses/by/ $4.0 /)$.

\begin{abstract}
The association of plasmonic nanostructures with nonlinear dielectric systems has been shown to provide useful platforms for boosting frequency conversion processes at metal-dielectric interfaces. Here, we report on an efficient route for engineering light-matter interaction processes in hybrid plasmonic- $\chi^{(2)}$ dielectric systems to enhance second harmonic generation (SHG) processes confined in small spatial regions. By means of ferroelectric lithography, we have fabricated scalable micrometric arrangements of interacting silver nanoparticles compactly distributed on hexagonal regions. The fabricated polygonal microstructures support both localized and extended plasmonic modes, providing large spatial regions of field enhancement at the optical frequencies involved in the SHG process. We experimentally demonstrate that the resonant excitation of the plasmonic modes supported by the Ag nanoparticle-filled hexagons in the near infrared region produces an extraordinary $10^{4}$-fold enhancement of the blue second harmonic intensity generated in the surface of a $\mathrm{LiNbO}_{3}$ crystal. The results open new perspectives for the design of efficient hybrid plasmonic frequency converters in miniaturized devices.
\end{abstract}

Keywords: metallic nanoparticles; second harmonic generation; hybrid plasmonic-dielectric; nanophotonics; nonlinear optics; hexagonal microcavity; lithium niobate

\section{Introduction}

The generation and manipulation of nonlinear optical processes at the nanoscale is a highly interesting field due to its broad range of applications in a variety of disciplines such as biology, physics, chemistry, materials science, and information technologies [1-3]. However, limitations associated with the low conversion efficiency at small scales require the use of novel designs to develop efficient nonlinear optical sources operating in extremely confined volumes. In the last decade, different approaches based on plasmon-driven light confinement have been used to enhance the nonlinear response of a variety of systems of reduced dimensions. In fact, the large near field enhancement provided by localized surface plasmon resonances (LSPRs) compensates for the lack of phase matching mechanisms at the nanoscale, leading to high nonlinear responses at subwavelength scales [4-6]. Depending on the size and geometry of the plasmonic nanostructures, the enhancement of the electromagnetic field can be achieved either at the harmonic or the fundamental frequency, the latter being much more efficient due to the nonlinear dependence of the generated harmonic signal with the fundamental radiation [7-10].

In recent years, the use of plasmonic nanostructures for light confinement in nonlinear dielectrics has emerged as an efficient and straightforward approach for boosting the frequency doubling response in miniaturized components. This approach exploits both the high nonlinear coefficients offered by certain nonlinear dielectric crystals and the strong light confinement provided by plasmonic structures, which has led to efficient 
hybrid plasmonic- $\chi^{(2)}$ dielectric systems operating at different spectral ranges. The proposed designs include core-shell nanoparticles (ferroelectric nanoparticles surrounded by $\mathrm{Au}$ shells [11,12]), semiconductors or nonlinear dielectrics in the gap between plasmonic nanoantennas [13-15], colloidal BTO-Au hybrid nano-dimmers [16], Au nanoring resonators filled with $\mathrm{LiNbO}_{3}$ [17], hybrid plasmonic waveguides [18,19], or ordered and disordered plasmonic arrangements of interacting silver nanoparticles (NPs) assembled on the polar surface of ferroelectric crystals [20-24].

In this work, we use an alternative approach to demonstrate SHG enhancement by around four orders of magnitude in $\mathrm{LiNbO}_{3}$ by means of interacting Ag NPs compactly distributed within polygonal surfaces onto a ferroelectric domain patterned nonlinear $\mathrm{LiNbO}_{3}$ crystal. $\mathrm{LiNbO}_{3}$ is one of the most widely employed dielectrics for advanced photonics and optoelectronics due to its remarkable acousto-optic, electro-optic, and nonlinear coefficients [25-27]. The association of $\mathrm{LiNbO}_{3}$ with noble metal plasmonic nanostructures has shown to be a useful route to provide solid-state platforms for boosting nonlinear processes at the nanoscale $[17,19,21,28]$. SHG enhancement factors as high as 400 have been reported by using hexagonal plasmonic necklaces of silver nanoparticles formed on the $\mathrm{LiNbO}_{3}$ crystal surface [21]. Additionally, theoretical works have pointed out the possibility of achieving giant $\mathrm{SHG}$ enhancements by using either Ag-coated $\mathrm{LiNbO}_{3}$ core-shell nanocuboids with designed double plasmonic resonances [29] or hybrid nanoplasmonic waveguides [30].

Here, we go a step further to experimentally demonstrate that SHG in $\mathrm{LiNbO}_{3}$ can be enhanced by around four orders of magnitude by completely filling the inside of the hexagonal necklaces with interacting silver NPs. The fabricated plasmonic structure provides two spectrally overlapping plasmonic resonances; namely, a collective localized plasmonic mode sustained by the surrounding hexagonal polygonal necklace (centered at around $535 \mathrm{~nm}$ ) and a spatially extended plasmonic mode centered at $705 \mathrm{~nm}$, which is associated with the LSPR of the Ag NPs covering the hexagonal regions. These Ag $\mathrm{Np}$ filled hexagons are responsible for an efficient enhancement of the fundamental NIR radiation, thus leading to giant SHG enhancement values of about $10^{4}$ in the blue spectral region due to the two-photon character of the nonlinear process. The results provide an alternative strategy to design cost-efficient and scalable plasmonic architectures to enhance SHG processes in hybrid plasmonic-dielectric systems, opening new opportunities for increasing the nonlinear conversion efficiency in small volumes.

The manuscript is structured as follows. First, we briefly describe the fabrication procedure and the main sample features. Then, the optical response of large-filled hexagons (5-10 $\mu \mathrm{m}$ side length) as well as their capability to boost the SHG in the nonlinear material are studied and compared to those obtained for empty hexagonal necklaces with similar dimensions. Finally, the influence of the hexagon size on the SHG enhancement is analyzed. The results show that by reducing the size of the filled hexagons to dimensions close to the fundamental radiation wavelength (size length of hexagons around $700 \mathrm{~nm}$ ), the nonlinear emission process is highly improved and an extraordinary $10^{4}$-fold enhancement of the blue second harmonic intensity generated in the surface of a $\mathrm{LiNbO}_{3}$ crystal is obtained. The results, related to the interconnection of the NPs structure and to the collective modes in the wavelength-scale hexagonal arrangements, validate the optical performance of the hybrid system in a spectral region of high technological interest.

\section{Materials and Methods}

\subsection{Sample Preparation}

Domain Fabrication: Two dimensional patterns of hexagonal ferroelectric domains were fabricated in a $0.5 \mathrm{~mm}$ thick $\mathrm{z}$-cut $\mathrm{LiNbO}_{3}$ single domain crystal by direct electron beam irradiation using a Philips XL30 SFEG electron microscope driven by an Elphy Raith nanolithography software. The beam current and acceleration voltage were fixed at $0.3 \mathrm{nA}$ and $15 \mathrm{kV}$, respectively. Additionally, during the irradiation process, the $z+$ face was coated with an $\mathrm{Al}$ film of $100 \mathrm{~nm}$ acting as ground electrode. After irradiation, scanning electron 
microscopy (SEM) microscopy was employed to confirm that the inverted hexagonal domains crossed the whole sample thickness, their size and shape being similar at both crystal faces [31,32].

Ag Decoration: The photo-deposition of metallic NPs was carried out by ferroelectric lithography by immersing the domain patterned crystal into a $0.01 \mathrm{M} \mathrm{AgNO}_{3}$ solution at $60{ }^{\circ} \mathrm{C}$ while their polar surface was illuminated with above band gap UV radiation with a mercury pen lamp (UVP model 11SC-1) with its main line at $253.6 \mathrm{~nm}$. The $\mathrm{Hg}$ lamp emission power was $5400 \mu \mathrm{W} / \mathrm{cm}^{2}$ at a distance of $2 \mathrm{~cm}$. The illumination time was varied to achieved different types of hybrid structures. More specifically, the formation of empty hexagonal necklaces was achieved for an illumination time of $4 \mathrm{~min}$ and the fabrication of Ag-filled hexagonal regions was achieved after illuminating the $\mathrm{AgNO}_{3}$ solution for $15 \mathrm{~min}$. In both cases, the photoinduced silver deposition process was carried out on optical grade polished crystals. Finally, it is worth mentioning that while the formation of hexagonal necklaces can be achieved illuminating the crystal at any polar face, the Ag-filled hexagonal regions correspond to ferroelectric domains with a $z+$ positive polarity. That is, the photoinduced silver deposition must be carried out by illuminating the $z-$ face of the crystal containing $z+$ hexagonal domains.

\subsection{Optical Characterization}

Dark field images were obtained in transmission configuration by using an Olympus BX51 microscope equipped with a dark-field condenser. For the extinction spectra, a double beam Lambda 1050 PerkinElmer spectrometer was employed.

Spatially resolved SHG experiments were carried out by means of a laser scanning confocal microscope (Olympus BX41) provided with a two-axis XY motorized platform driven by commercial Labspec software. The spatial resolution of the stage was $0.3 \mu \mathrm{m}$. The fundamental beam, delivered by a femtosecond pulsed Ti:sapphire laser (Spectra Physics Model 177-Series), was tuned at $850 \mathrm{~nm}$ and focused onto the sample surface by a $100 \times$ microscope objective. The SHG signal was collected in backscattering geometry with the same objective and detected by a Peltier cooled photomultiplier tube.

\section{Results and Discussion}

\subsection{Fabrication Process. Sample Characteristics}

Ag NPs assembled on hexagonal arrangements were obtained on the polar surface of $\mathrm{LiNbO}_{3}$ crystals by using hexagonal inverted ferroelectric domains as artificial templates for selective photo-deposition process [21,22]. The side length of the hexagons varied from $0.7 \mu \mathrm{m}$ up to $10 \mu \mathrm{m}$, in such a way that the contour of a single hexagon consisted of a necklace containing hundreds of Ag NPs. The domain selective formation of Ag NPs on a positive domain is based on the photo-chemical reactions that take place on the surface of domain patterned ferroelectric crystals [33,34]. More specifically, when the patterned ferroelectric is placed into an $\mathrm{AgNO}_{3}$ solution and illuminated with aboveband-gap UV light, the screening charge produces electronic photocarriers at the crystal surface, which participate in the chemical reaction reducing the silver cations on the specific polarity domains [34]. In the case of $\mathrm{LiNbO}_{3}, \mathrm{Ag} \mathrm{NP}$ formation involves both a weak downward band bending at positive domain surfaces and a strong electric field at domain boundaries [35]. This allows a certain degree of control over the process so that the Ag deposition on the domain surface and the deposition on the domain boundary surface can be achieve independently. Accordingly, by modifying the illumination time, either Ag NPs hexagonal necklaces formed on the domain wall boundaries or a dense distribution of closely spaced interacting Ag nanoparticle covering the positive domain surface can be obtained. Specific details on the preparation method can be found elsewhere [22].

Figure 1 shows SEM images of the resultant metallic nanostructures after different photo-deposition processes. By varying the illumination time while keeping constant the rest of the parameters $\left(0.01 \mathrm{M} \mathrm{AgNO}_{3}\right.$ solution, $5400 \mu \mathrm{W} / \mathrm{cm}^{2} \mathrm{UV}$ emission power, $60{ }^{\circ} \mathrm{C}$ substrate temperature) different type of plasmonic arrangements are obtained on 
the polar surface of $\mathrm{LiNbO}_{3}$. Figure 1a shows a hexagonal plasmonic necklace of Ag NPs on the domain boundary surface of $\mathrm{LiNbO}_{3}$ obtained for an illumination time of $4 \mathrm{~min}$. As observed, the chain of Ag NPs is perfectly located on the domain boundary surface of a hexagonal ferroelectric domain, bending at vertices to form a polygonal necklace (Figure 1b). According to the distribution displayed in Figure 1c, the average size of the Ag NPs forming the hexagonal necklace is around $50 \mathrm{~nm}$ and the interparticle distance about $2 \mathrm{~nm}$.
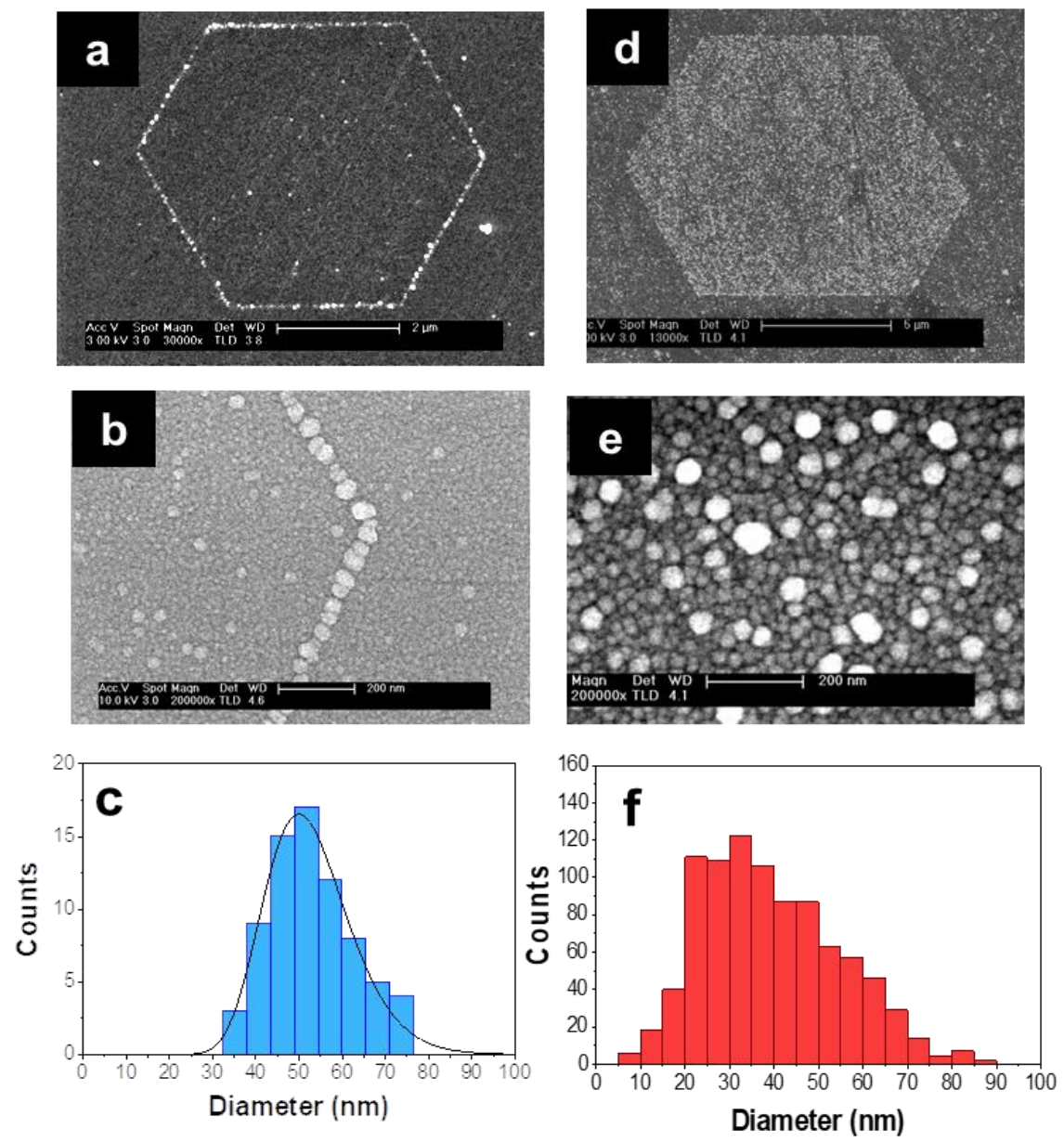

Figure 1. Plasmonic hexagonal arrangements on $\mathrm{LiNbO}_{3}$ substrate. SEM images of (a) Hexagonal plasmonic necklace formed on the domain boundary surface of $\mathrm{LiNbO}_{3}$ at a photo-deposition time of 4 min. (b) Detailed view of the Ag NPs forming the vertex of a hexagonal necklace. (c) Histogram of the Ag NPs diameter forming the necklace. (d) Filled hexagonal domain. It is formed by a dense distribution of Ag NPs covering the positive domain surface after a photo-deposition time of $15 \mathrm{~min}$. (e) Detail of the Ag NPs that cover a positive ferroelectric domain of hexagonal shape. (f) Histogram of the Ag NPs diameter covering the filled hexagonal region.

Increasing the illumination time leads to the formation of Ag NPs that completely cover the surface of positive ferroelectric domains after $15 \mathrm{~min}$ of illumination. This assembling of nearby NPs within the hexagon provides a straightforward fabrication of large spatial regions of high density of interacting plasmonic NPs confined inside a specific polygonal zone (Figure 1d). A detailed image of the Ag NPs located inside the plasmonic hexagon is shown in Figure 1e. As can be seen, a high density of mostly spherical Ag NPs fills the polygonal region. Although in this case, a slightly larger size dispersion is obtained (Figure 1f), the average NP size is similar to that of the Ag NPs necklaces. Additionally, comparable values of the interparticle distance are obtained in both type of Ag NPs arrangements (around $2 \mathrm{~nm}$ ). Finally, it is worth mentioning that the fabricated 
polygonal microstructures can be organized in two-dimensional superlattices leading to scalable functional nonlinear metasurfaces [36].

\subsection{Optical Response of Filled Polygonal Assemblies}

The dark-field scattering image of both types of arrangements is displayed in Figure 2. In both cases, the radiative nature of the plasmonic modes is observed. For the filled hexagons, a predominantly reddish color spreads throughout the decorated domain surface (Figure 2a). Differently, the dark field image associated with the hexagonal necklaces (short photo-deposition times) shows a dominant orange color, the scattered light being produced along the contour of the necklace where the Ag NPs are located. The corresponding extinction spectra obtained in transmission mode after subtracting the contribution from the bare $\mathrm{LiNbO}_{3}$ substrate are depicted in Figure 2b. As seen, in both cases a broad resonance with the maximum around $\lambda_{I}=535 \mathrm{~nm}$ is observed. In the case of the Ag NPs hexagonal necklaces, the spectrum corresponds to the collective mode sustained by the necklaces as previously reported by the authors [21,37]. In the case of the filled hexagons, the additional spectral band appearing at the low energy side (centered at around $\lambda_{\mathrm{II}}=705 \mathrm{~nm}$ ) can be related to the highly dense Ag NPs distribution decorating the interior of the hexagon. Both the $\lambda_{\mathrm{I}}$ and $\lambda_{\mathrm{II}}$ values correspond to the central wavelength of two Gaussian profiles that fit the extinction spectrum of the plasmonic structures shown in Figure 2b.
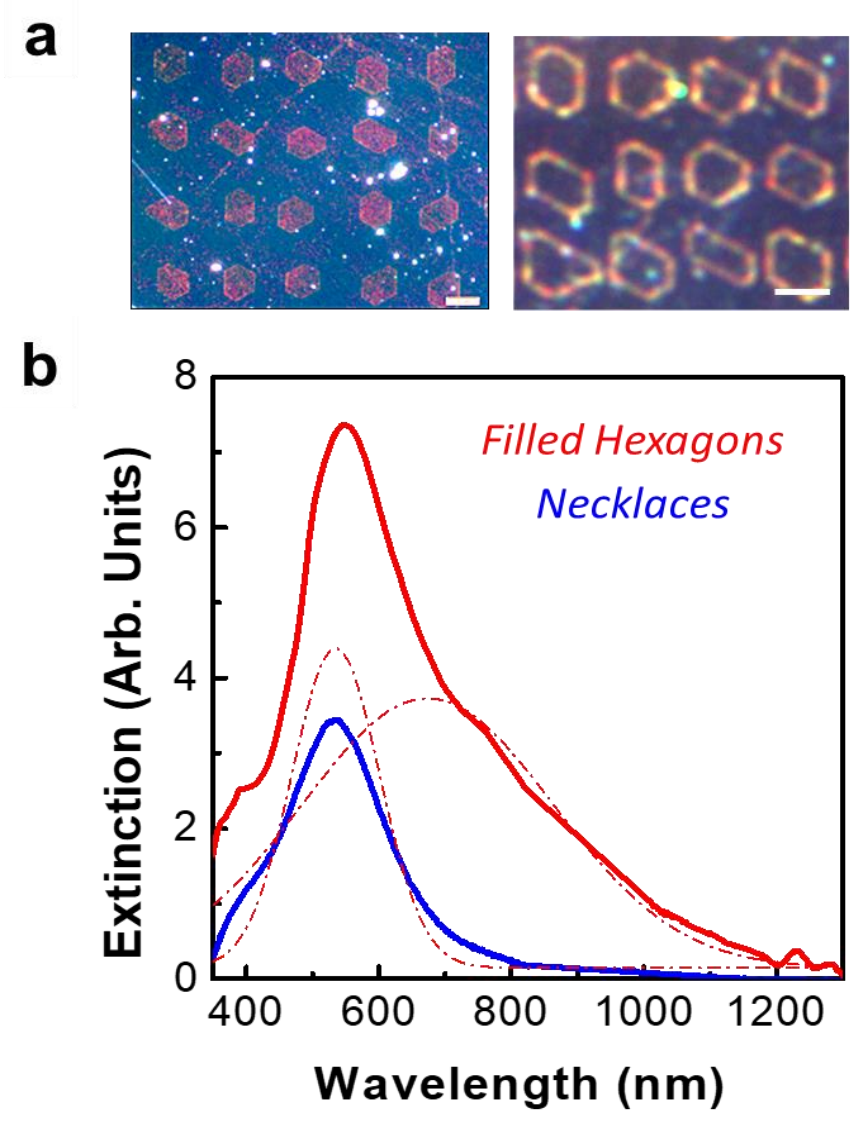

Figure 2. Far field optical response of the polygonal assemblies. (a) Dark-field scattering images recorded for the two types of Ag NPs hexagonal arrangement. Left: filled hexagons, Right: hexagonal necklaces. The scale bar is $5 \mu \mathrm{m}$. (b) Extinction spectra obtained in transmission mode after subtracting the contribution from the bare $\mathrm{LiNbO}_{3}$ substrate. Blue: hexagonal necklaces. Red: filled hexagons. The dashed red lines correspond to Gaussian fitting functions.

The appearance of the low energy band can be explained in the frame of previous studies, which show that complex NP structures can be efficiently analyzed by an embed- 
ded chain plasmon model [38-40]. In this context, the spectral response of the complex plasmonic structure filling the hexagons can be interpreted as the contribution of a variety of linear or quasi-linear kinked NP chains within the hexagons. As a result, numerous plasmonic chains, with different numbers of interacting NPs, contribute to the total spectral response in the far field. Moreover, significant chain coupling has been reported for chain separation distances of a few nanometers, which shifts the resonance to the near infrared region [41]. This chain plasmon mode has been shown to be useful to enhance a variety of optical phenomena including SHG, SERS or nanolasing, because of its robustness, strong radiative nature, and broad spectral response [21,42,43]. On the other hand, the assembly of NPs inside the hexagons also shows certain similarity to percolated systems or disordered plasmonic networks where a spectral shift of the plasmonic resonance towards the near infrared spectral region has been reported due to the presence of NP clusters extended in relatively large spatial regions [44-46].

The effect of the plasmonic arrangements on the SHG response generated by the nonlinear substrate was analyzed by means of confocal scanning microscopy. The experiments were performed at a fundamental wavelength of $850 \mathrm{~nm}$, which efficiently overlaps the low energy side of the spectra shown Figure 2. The $\mathrm{LiNbO}_{3}$ substrate also exhibits a good transparency range for this wavelength, enabling the generation of SHG in the blue spectral region, which is of technological interest in the context of potential nonlinear photonic nanodevices. Figure 3 shows the spatial distribution of the blue SHG signal generated in the $\mathrm{LiNbO}_{3}$ substrate, which is enhanced by the plasmonic nanostructures. The spatial maps displayed in Figure 3a show that the SHG signal is greatly boosted in the presence of the metallic nanostructures with respect to that of the bare substrate, reproducing the spatial distribution of the Ag nanoparticles. That is, the SHG enhancement remains localized at the domain boundary surfaces in the case of hexagonal necklaces and extends over the whole domain surface in the case of the Ag NP filled domains. For illustrative purposes, the SHG spatial maps were normalized to their maximum values. The cross-section intensity profiles depicted in Figure $3 \mathrm{~b}$ show the quantitative comparison of the SHG enhancement in both systems. As can be seen, the enhanced SHG intensity shows remarkable differences when comparing the response of both types of structures, the improved nonlinear response in the filled hexagons being two orders of magnitude greater than that obtained from the necklaces.

To estimate the enhancement factor, we compared the nonlinear performance of the hybrid architectures with respect to that generated in the substrate in the absence of metallic NPs. To that end, we experimentally determined the fundamental pump power required to achieve a comparable SHG intensity. The results obtained for the three analyzed systems were: $26 \mathrm{~mW}$ for the bare $\mathrm{LiNbO}_{3}$ substrate, $3 \mathrm{~mW}$ for the hybrid $\mathrm{LiNbO}_{3}$-plasmonic necklaces, and $1 \mathrm{~mW}$ for the filled hexagons. Hence, according to the quadratic dependence of the SHG response on the fundamental power, an SHG enhancement value of 675 is obtained for the filled hexagons, while an enhancement factor of 9 is achieved for the hexagonal necklaces. Figure $3 c$ compares in log scale the enhancement factors obtained from both types of plasmonic structures. The dramatic difference between them can be accounted for by the presence of the low energy plasmonic resonance associated with the filled hexagons (see Figure 2), which provides a much more efficient spectral overlap of the fundamental radiation with respect to the case of the hexagonal necklaces. Accordingly, a significantly larger SHG enhancement due to the two-photon character of the frequency conversion process is obtained.

Finally, we should emphasize that the SHG signal is generated in the $\mathrm{LiNbO}_{3}$ nonlinear substrate. This fact was confirmed by analyzing the SHG intensity as a function of the polarization of the fundamental radiation. The resulting polar plots of SHG intensity for the bare substrate and for the Ag NPs filled hexagon obtained in reflection geometry are shown in Figure 3d. For the sake of comparison, the nonlinear signal in the absence of Ag NPs was registered with an incident pump power 20 times greater than the one employed for the filled hexagon $(\sim 1 \mathrm{~mW})$. As observed, they show similar trends and both polar plots are consistent with the $3 \mathrm{~m}$ point group symmetry of $\mathrm{LiNbO}_{3}$. Therefore, we can conclude 
that the $\mathrm{SH}$ signal is generated in the nonlinear $\mathrm{LiNbO}_{3}$ crystal, the contribution of the $\mathrm{Ag}$ nanostructures to the SHG being negligible.

a
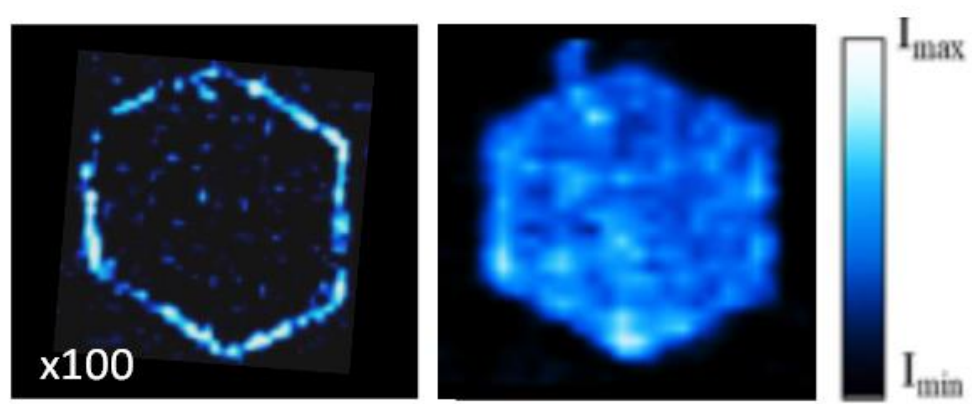

b

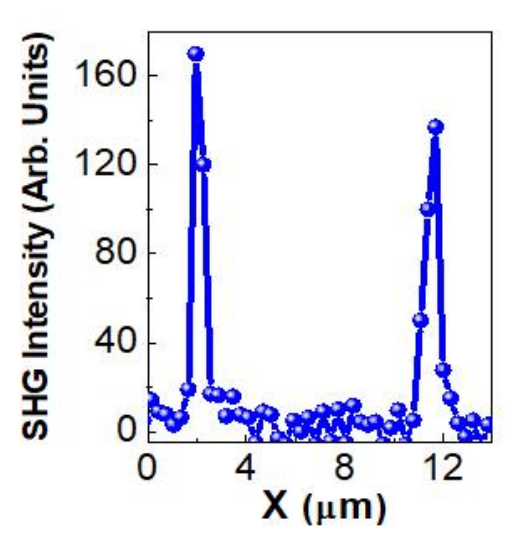

C

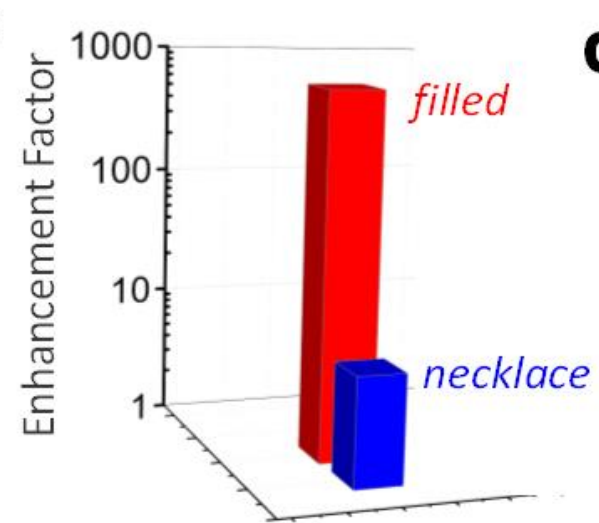

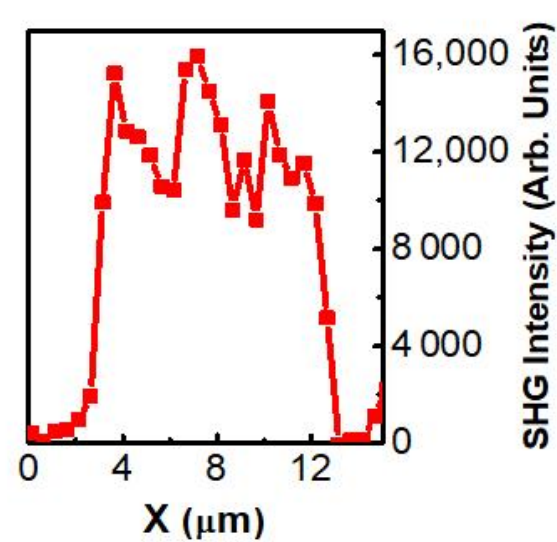

d

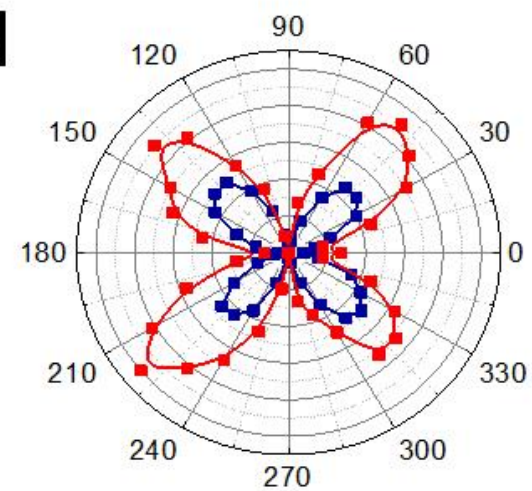

Figure 3. SHG enhancement: Filled hexagons vs hexagonal necklaces. (a) Spatial distribution of the SHG intensity generated in the $\mathrm{LNbO}_{3}$ substrate in the proximities of an Ag NPs hexagonal necklace (left) and Ag NPs filled hexagon (right) for a fundamental radiation wavelength of $850 \mathrm{~nm}$. The SHG intensity map associated with the necklaces is multiplied by a factor of 100 . The scale bar corresponds to $5 \mu \mathrm{m}$. (b) SHG intensity profile obtained from the SHG maps shown in (a). The fundamental pump power was about $1 \mathrm{~mW}$. (c) Comparison between the SHG enhancement factor for the filled hexagon (red) and for the hexagonal necklace (blue). (d) Variation of the polarized SHG intensity as a function of the polarization angle of the incident radiation for filled hexagons (red) and bare $\mathrm{LiNbO}_{3}$ (dark blue).

\subsection{Scaling Effects on SHG Enhancement}

Once the capability of large-filled hexagons to enhance the SHG process has been demonstrated, we aim to study the effect of reducing the hexagon size to dimensions comparable to that of the fundamental radiation wavelength. Figure 4a illustrates the SHG obtained for a distribution of hexagonal domains with side lengths around $700 \mathrm{~nm}$. A striking difference is observed when comparing the enhancement factors obtained for 
large and small hexagons (Figure $4 \mathrm{~b}$ ). In contrast to the 670 -fold value featured by the large-filled hybrid hexagons, the enhancement factor obtained for small hexagons is close to $10^{4}$. Indeed, the SHG spatial maps in these structures were recorded with an incident pump power of about $0.15 \mathrm{~mW}$, that is one order of magnitude lower than that used for the large hexagons.

a
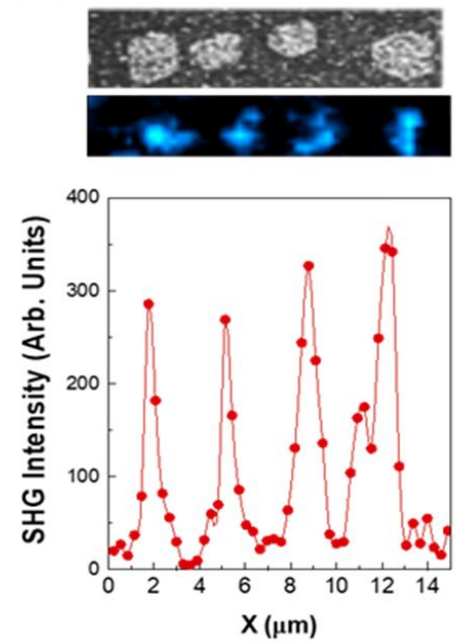

b

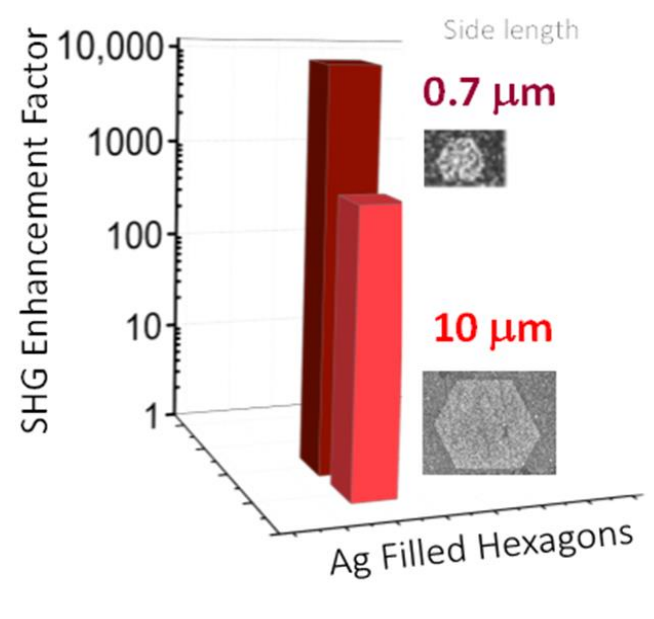

Figure 4. SHG enhancement by small-filled hexagons. (a) Top panel: SEM image and SHG spatial map from a region comprising four hexagons (hexagon sides around $700 \mathrm{~nm}$ ). The blue regions in the map correspond to the SHG intensity. Bottom panel: SHG intensity area across the hexagons. (b) SHG enhancement factor for Ag NPs filled hexagons with hexagon sides of about $10 \mu \mathrm{m}$ (red) and $700 \mathrm{~nm}$ (dark red).

The much larger enhancement in the small hexagons could be related to the existence of extended collective plasmonic modes propagating through the entire hexagon. In fact, the possibility of using the inner NP structure as plasmonic interconnection between the different sides of the hexagon could also favor the propagation of the plasmon mode inside the hexagon [47-51]. Additionally, due to the hexagon dimensions, the presence of modes in the wavelength-scale hexagonal nanocavity could also contribute to the light confinement and enhanced light-matter interaction process [52].

\section{Summary and Conclusions}

To summarize, we have presented an alternative hybrid plasmonic-nonlinear dielectric system for enhancing SHG processes at reduced dimensions. Using hexagonal plasmonic necklaces filled with a dense distribution of interacting Ag NPs, we show that the second harmonic response generated from the surface of $\mathrm{LiNbO}_{3}$ crystal can be enhanced in about four orders of magnitude. Such a large enhancement is achieved by taking advantage of the strong near field generated at the metal-dielectric interface associated with the resonant excitation of localized and spatially extended plasmonic modes in small filled hexagons. The ability to combine both plasmonic fields in a single microstructure and the possibility to resonantly excite them by means of the optical fields involved in the nonlinear process, provides an efficient route for engineering light-matter interaction processes at subwavelength scales to enhance parametric nonlinear processes. Because of the ease of the fabrication process and the extraordinary improvement of the SHG response in small-confined region, our findings offer an alternative approach for the design of efficient hybrid plasmonic frequency converter nanodevices and opens interesting perspectives for a range of applications including sensing, imaging, information technologies, or future integrated circuits. 
Author Contributions: Conceptualization, M.O.R.; methodology and investigation A.G.-T.; validation, A.G.-T., L.E.B. and M.O.R.; formal analysis, A.G.-T., L.E.B. and M.O.R.; supervision, M.O.R.; All authors have read and agreed to the published version of the manuscript.

Funding: This work has been supported by the Spanish Government (contracts MAT2016-76106-R and PID2019-108257GB-I00 and María de Maeztu "Programme for Units of Excellence in R\&D" CEX2018-000805-M).

Data Availability Statement: Data underlying the results presented in this paper are not publicly available at this time but may be obtained from the authors upon reasonable request.

Conflicts of Interest: The authors declare no conflict of interest.

\section{References}

1. Ray, P.C. Size and shape dependent second order nonlinear optical properties of nanomaterials and their application in biological and chemical sensing. Chem. Rev. 2010, 110, 5332-5365. [CrossRef] [PubMed]

2. Wang, K.; Titchener, J.G.; Kruk, S.S.; Xu, L.; Chung, H.-P.; Parry, M.; Kravchenko, I.I.; Che, Y.-H.; Solntsev, S.S.; Kivshar, Y.S.; et al. Quantum metasurface for multiphoton interference and state reconstruction. Science 2018, 361, 1104-1108. [CrossRef]

3. Butet, J.; Russier-Antoine, I.; Jonin, C.; Lascoux, N.; Benichou, E.; Brevet, P.F. Sensing with multipolar second harmonic generation from spherical metallic nanoparticles. Nano Lett. 2012, 12, 1697-1701. [CrossRef] [PubMed]

4. Bonacina, L.; Brevet, P.F.; Finazzi, M.; Celebrano, M. Harmonic generation at the nanoscale. J. Appl. Phys. 2020, $127,230901$. [CrossRef]

5. Kauranen, M.; Zayats, A. Nonlinear plasmonics. Nat. Photonics 2012, 6, 737-748. [CrossRef]

6. Butet, J.; Brevet, P.F.; Martin, O.J.F. Optical second harmonic generation in plasmonic nanostructures: From fundamental principles to advanced application. ACS Nano 2015, 9, 10545-10562. [CrossRef]

7. Celebrano, M.; Wu, X.F.; Baselli, M.; Großmann, S.; Biagioni, P.; Locatelli, A.; De Angelis, C.; Cerullo, G.; Osellame, R.; Hecht, B.; et al Mode matching in multiresonant plasmonic nanoantennas for enhanced second harmonic generation. Nat. Nanotechnol. 2015, 10, 412-417. [CrossRef]

8. Weber, N.; Protte, M.; Walter, F.; Georgi, P.; Zentgraf, T.; Meier, C. Double resonant plasmonic nanoantennas for efficient second harmonic generation in zinc oxide. Phys. Rev. B 2017, 95, 205307. [CrossRef]

9. Thyagarajan, K.; Rivier, S.; Lovera, A.; Martin, O.J.F. Enhanced second-harmonic generation from double resonant plasmonic antennae. Opt. Express 2012, 12, 12860-12865. [CrossRef] [PubMed]

10. Yang, K.Y.; Butet, J.; Yan, C.; Bernasconi, G.D.; Martin, O.J.F. Enhancement mechanisms of the second harmonic generation from double resonant aluminum nanostructures. ACS Photonics 2017, 4, 1522-1530. [CrossRef]

11. Pu, Y.; Grange, R.; Hsieh, C.L.; Psaltis, D. Nonlinear Optical Properties of Core-Shell Nanocavities for Enhanced Second-Harmonic Generation. Phys. Rev. Lett. 2010, 104, 207402. [CrossRef] [PubMed]

12. Richter, J.; Steinbrück, A.; Zilk, M.; Sergeyev, A.; Pertsch, T.; Tünnermann, A.; Grange, R. Core-shell potassium niobate nanowires for enhanced nonlinear optical effects. Nanoscale 2014, 6, 5200-5207. [CrossRef]

13. Chauvet, N.; Maeliss, E.; Jeannin, M.; Laurent, G.; Huant, S.; Gacoin, T.; Dantelle, G.; Nogues, G.; Bachelier, G. Hybrid KTP-Plasmonic Nanostructures for Enhanced Nonlinear Optics at the Nanoscale. ACS Photonics 2020, 7, 665-672. [CrossRef]

14. Metzger, B.; Hentschel, M.; Schumacher, T.; Lippitz, M.; Ye, X.; Murray, C.B.; Knabe, B.; Buse, K.; Giessen, H. Doubling the efficiency of third harmonic generation by positioning ITO nanocrystals into the hot-spot of plasmonic gap-antennas. Nano Lett. 2014, 14, 2867-2872. [CrossRef] [PubMed]

15. Linnenbank, H.; Grynko, Y.; Forstner, J.; Linden, S. Second harmonic generation spectroscopy on hybrid plasmonic/dielectric nanoantennas. Light Sci. Appl. 2016, 5, e16013. [CrossRef]

16. Timpu, F.; Hendricks, N.R.; Petrov, M.; Ni, S.; Renaut, C.; Wolf, H.; Isa, L.; Kivshar, Y.; Grange, R. Enhanced Second-Harmonic Generation from Sequential Capillarity-Assisted Particle Assembly of Hybrid Nanodimers. Nano Lett. 2017, 17, 5381-5388. [CrossRef]

17. Lehr, D.; Reinhold, J.; Thiele, I.; Hartung, H.; Dietrich, K.; Menzel, C.; Pertsch, T.; Kley, E.-B.; Tünnermann, A. Enhancing Second Harmonic Generation in Gold Nanoring Resonators Filled with Lithium Niobate. Nano Lett. 2015, 15, 1025-1030. [CrossRef] [PubMed]

18. Shi, J.J.; Li, Y.; Kang, M.; He, X.; Halas, N.J.; Nordlander, P.; Zhang, S.; Xu, H. Efficient second harmonic generation in a hybrid plasmonic waveguide by mode interactions. Nano Lett. 2019, 19, 3838-3845. [CrossRef]

19. Li, Z.; Corbett, B.; Gocalinska, A.; Pelucchi, E.; Chen, W.; Ryan, K.M.; Khan, P.; Silien, C.; Xu, H.; Liu, N. Direct Visualization of Phase-Matched Efficient Second Harmonic and Broadband Sum Frequency Generation in Hybrid Plasmonic Nanostructures. Light Sci. Appl. 2020, 9, 180. [CrossRef]

20. Yraola, E.; Molina, P.; Plaza, J.L.; Ramírez, M.O.; Bausá, L.E. Spontaneous emission and nonlinear response enhancement by silver nanoparticles in a Nd3+doped periodically poled $\mathrm{LiNbO}_{3}$ laser crystal. Adv. Mater. 2013, 25, 910-915. [CrossRef]

21. Gomez-Tornero, A.; Tserkezis, C.; Mateos, L.; Bausá, L.E.; Ramírez, M.O. 2D Arrays of Hexagonal Plasmonic Necklaces for Enhanced Second Harmonic Generation. Adv. Mater. 2017, 29, 160527. [CrossRef] 
22. Ramírez, M.O.; Molina, P.; Gómez-Tornero, A.; Hernández-Pinilla, D.; Sánchez-García, L.; Carretero, S.; Bausá, L.E. Hybrid Plasmonic-Ferroelectric Architectures for Lasing and SHG Processes at the Nanoscale. Adv. Mater. 2019, 31, 1901428. [CrossRef] [PubMed]

23. Sánchez-García, L.; Ramírez, M.O.; Molina, P.; Gallego-Gómez, F.; Mateos, L.; Yraola, E.; de las Heras, C.; Bausá, L.E. Blue SHG Enhancement by Silver Nanocubes Photochemically Prepared on $\mathrm{RbTiOPO}_{4}$ Ferroelectric Crystal. Adv. Mater. 2014, $26,6447$. [CrossRef] [PubMed]

24. Sánchez-García, L.; Tserkezis, C.; Ramírez, M.O.; Molina, P.; Carvajal, J.J.; Aguiló, M.; Díaz, F.; Aizpurua, L.; Bausá, L.E. Plasmonic enhancement of second harmonic generation from nonlinear $\mathrm{RbTiOPO}_{4}$ crystals by silver nanoaggregates. Opt. Express 2016, 24, 8491-8500. [CrossRef] [PubMed]

25. Lin, J.; Bo, F.; Cheng, Y.; Xu, J. Advances in On-Chip Photonic Devices Based on Lithium Niobate on Insulator. Photonics Res. 2020, 8, 1910-1936. [CrossRef]

26. Boes, A.; Corcoran, B.; Chang, L.; Bowers, J.; Mitchell, A. Status and Potential of Lithium Niobate on Insulator (LNOI) for Photonic Integrated Circuits. Laser Photonics Rev. 2018, 12, 1700256. [CrossRef]

27. Sun, D.; Zhang, Y.; Wang, D.; Song, W.; Liu, X.; Pang, J.; Geng, D.; Sang, Y.; Liu, H. Microstructure and Domain Engineering of Lithium Niobate Crystal Films for Integrated Photonic Applications. Light Sci. Appl. 2020, 9, 197. [CrossRef]

28. Gürdal, E.; Horneber, A.; Meixner, A.J.; Kern, D.P.; Zhang, D.; Fleischer, M. Enhancement of the second harmonic signal of nonlinear crystals by a single metal nanoantenna. Nanoscale 2020, 12, 23105-23115. [CrossRef]

29. Ren, M.; Liu, S.; Wang, B.; Chen, B.; Jiafan, L.; Yuan, Z. Gian enhancement of second harmonic by engineering double plasmonic resonances at nanoscale. Opt. Express 2014, 22, 28653-28661. [CrossRef]

30. Carnio, B.N.; Elezzabi, A.Y. Second harmonic generation in metal-LiNbO 3 -metal and $\mathrm{LiNbO}_{3}$ hybrid-plasmonic waveguides. Opt. Express 2018, 26, 26283-26291. [CrossRef]

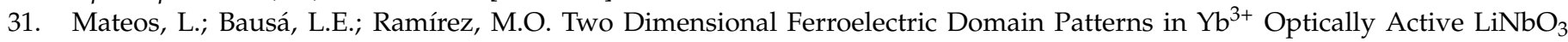
Fabricated By Direct Electron Beam Writing. Appl. Phys. Lett. 2013, 102, 042910. [CrossRef]

32. Mateos, L.; Bausá, L.E.; Ramírez, M.O. Micro-spectroscopic characterization of ferroelectric domain structures in $\mathrm{Yb}^{3+}: \mathrm{LiNbO}_{3}$ prepared by electron beam writing. Opt. Mater. Express 2014, 4, 1077-1087. [CrossRef]

33. Kalinin, S.V.; Bonnell, D.A.; Alvarez, T.; Lei, X.J.; Hu, Z.H.; Shao, R.; Ferris, J.H. Ferroelectric Lithography of Multicomponent Nanostructures. Adv. Mater. 2004, 16, 795-799. [CrossRef]

34. Kalinin, S.V.; Bonnell, D.A.; Alvarez, T.; Lei, X.; Hu, Z.; Ferris, J.H.; Zhang, Q.; Dunn, S. Atomic Polarization and Local Reactivity on Ferroelectric Surfaces: A New Route toward Complex Nanostructures. Nano Lett. 2002, 2, 589-593. [CrossRef]

35. Sun, Y.; Nemanich, R.J. Photoinduced Ag deposition on periodically poled lithium niobate: Wavelength and polarization screening dependence. J. Appl. Phys. 2011, 109, 104302. [CrossRef]

36. Gómez-Tornero, A.; Palacios, P.; Molina, P.; Carretero-Palacios, S.; Bausá, L.E.; Ramírez, M.O. Enhancing Nonlinear Interactions by the Superposition of Plasmonic Lattices on $\chi^{(2)}$-Nonlinear Photonic Crystals. ACS Photonics 2021, 8, 2529-2537. [CrossRef]

37. Gómez-Tornero, A.; Tserkezis, C.; Robledo-Moreno, J.; Bausá, L.E.; Ramírez, M.O. Field Enhancement and Spectral Features of Hexagonal Necklaces of Silver Nanoparticles for Enhanced Nonlinear Optical Processes. Opt. Express 2018, 26, 22394-22404. [CrossRef]

38. Taylor, R.W.; Esteban, R.; Mahajan, S.; Aizpurua, J.; Baumberg, J.J. Optimizing SERS from Gold Nanoparticle Clusters: Addressing the Near Field by an Embedded Chain Plasmon Model. J. Phys. Chem. C 2016, 120, 10512-10522. [CrossRef]

39. Esteban, R.; Taylor, R.W.; Baumberg, J.J.; Aizpurua, J. How chain plasmons govern the optical response in strongly interacting self-assembled metallic clusters of nanoparticles. Langmuir 2012, 28, 8881-8890. [CrossRef] [PubMed]

40. Tserkezis, C.; Taylor, R.W.; Beitner, J.; Esteban, R.; Baumberg, J.J.; Aizpurua, J. Optical response of metallic nanoparticle heteroaggregates with subnanometric gaps. Part. Part. Syst. Charact. 2014, 31, 152-160. [CrossRef]

41. Hanske, C.; Tebbe, M.; Kuttner, C.; Bieber, V.; Tsukruk, V.V.; Chanana, M.; Konig, T.A.F.; Fery, A. Strongly Coupled Plasmonic Modes on Macroscopic Areas via Template-Assisted Colloidal Self-Assembly. Nano Lett. 2014, 14, 6863-6871. [CrossRef]

42. Taylor, R.W.; Lee, T.; Scherman, O.A.; Esteban, R.; Aizpurua, J.; Huang, F.; Baumberg, J.J.; Mahaja, S. Precise Subnanometer Plasmonic Junctions for SERS within Gold Nanoparticle Assemblies using Cucurbit[n]uril “Glue". ACS Nano 2011, 5, 3878-3887. [CrossRef]

43. Molina, P.; Yraola, E.; Ramírez, M.O.; Tserkezis, C.; Plaza, J.L.; Aizpurua, J.; Bravo-Abad, J.; Bausá, L.E. Plasmon-Assisted $\mathrm{Nd}^{3+}$-based Solid-State Nanolaser. Nano Lett. 2016, 16, 895-899. [CrossRef]

44. Earp, A.A.; Smith, G.B. Evolution of plasmonic response in growing silver thin films with pre-percolation non-local conduction and emittance drop. J. Phys. D Appl. Phys. 2011, 44, 255102. [CrossRef]

45. Sánchez-García, L.; Ramírez, M.O.; Tserkezis, C.; Sole, R.; Carvajal, J.J.; Aguiló, M.; Díaz, F.; Bausá, L.E. Anisotropic enhancement of Yb ${ }^{3+}$ luminescence by disordered plasmonic networks self-assembled on $\mathrm{RbTiOPO}_{4}$ ferroelectric crystal. Nanoscale 2017, 9, 16166. [CrossRef] [PubMed]

46. Shalaev, M.; Ying, C.X.; Zhang, Z.; Cao, H. Coexistence of Localized and Delocalized Surface Plasmon Modes in Percolating Metal Films. Phys. Rev. Lett. 2006, 97, 206103.

47. Greybush, N.J.; Liberal, I.; Malassis, L.; Kikkawa, J.M.; Engheta, N.; Murray, C.B.; Kagan, C.R. Plasmon Resonances in SelfAssembled Two-Dimensional Au Nanocrystal Metamolecules. ACS Nano 2017, 11, 2917-2927. [CrossRef] 
48. Lee, S.; Sim, K.; Moon, S.; Choi, J.; Jeon, Y.; Nam, J.; Park, S.-J. Controlled Assembly of Plasmonic Nanoparticles: From Static to Dynamic Nanostructures. Adv. Mater. 2021, 2007668. [CrossRef] [PubMed]

49. Borah, R.; Verbruggen, W. Coupled Plasmon Modes in 2D Gold Nanoparticle Clusters and Their Effect on Local Temperature Control. J. Phys. Chem. C 2019, 123, 30594-30603. [CrossRef]

50. Luk'yanchuk, B.; Zheludev, N.I.; Maier, S.A.; Halas, N.J.; Nordlander, P.; Giessen, H.; Chong, C. The Fano resonance in plasmonic nanostructures and metamaterials. Nat. Mater. 2010, 9, 707-715. [CrossRef]

51. Phan, H.T.; Heiderscheit, T.S.; Haes, A.J. Understanding Time-Dependent Surface-Enhanced Raman Scattering from Gold Nanosphere Aggregates Using Collision Theory. J. Phys. Chem. C 2020, 124, 14287-14296. [CrossRef] [PubMed]

52. Yan, Y.; Tang, M.; Wang, F.L.; Xiao, Z.Z.; Xiao, J.L.; Huang, Y.Z. Whispering-gallery mode hexagonal micro-/nanocavity lasers. Photonics Res. 2019, 7, 594-607. 